\title{
Aktualisierte Richtlinien der Verkehrskommission der Schweizerischen Liga gegen Epilepsie (SLgE) \\ Epilepsie und Fahrtauglichkeit
}

\section{Verkehrskommission der SLgE*}

* Günter Krämer (Vorsitzender), Zürich; Claudio Bonetti,

Mendrisio; Paul-André Despland, Lausanne; Roland Markoff, Chur; Johannes Mathis, Bern; Vinicio Medici, Spiegel b. Bern; Klaus Meyer, Tschugg; Rolf Seeger, Zürich; Heinz-Gregor Wieser, Zürich
Korrespondenz:

Dr. med. Günter Krämer

Medizinischer Direktor

Schweizerisches Epilepsie-Zentrum

Bleulerstrasse 60

CH-8008 Zürich

Tel. 0443876302

Fax 0443876396

g.kraemer@swissepi.ch

\section{Einleitung}

Die Verkehrskommission der Schweizerischen Liga gegen Epilepsie (SLgE) hat zuletzt 1995 in Abstimmung mit der Schweizerischen Neurologischen Gesellschaft und Schweizerischen Gesellschaft für klinische Neurophysiologie überarbeitete Richtlinien zur Kraftfahrtauglichkeit bei Epilepsie publiziert [1]. Diese Richtlinien haben sich bewährt, und grundlegende Änderungen waren nicht erforderlich. Erfahrungen bei der praktischen Anwendung sowie kleinere Änderungen bei den Führerausweiskategorien waren jedoch Anlass für eine Aktualisierung mit einigen Präzisierungen und Ergänzungen.

Die wiederum mit den beiden obengenannten Fachgesellschaften abgestimmte Neufassung entspricht weitgehend den in anderen europäischen Staaten üblichen Regelungen [2, 3]. Neu gilt für langjährig anfallsfreie Patienten mit einem einzelnen Anfallsrezidiv eine verkürzte Karenzfrist. Ausserdem findet sich in der Anlage der Vorschlag für ein fachärztlich-neurologisches Zeugnisformular, das gegenüber den Strassenverkehrsämtern eine einheitliche Berichterstattung erleichtern soll.

Ein kürzlich von der Internationalen Liga gegen Epilepsie und dem Internationalen Büro für Epilepsie publizierter Vorschlag einer neuen Definition der Epilepsie, die danach unter bestimmten Voraussetzungen schon nach einem ersten Anfall gestellt werden kann [4], bleibt hier unberücksichtigt. Es sei auch betont, dass die hier vorgelegten Richtlinien dem Neurologen bewusst Spielraum für individuelle Abweichungen von den genannten Fristen lassen, sofern diese nachvollziehbar begründet werden können. In Abhängigkeit von weiterhin erforderlichen Harmonisierungen auf europäischer [5] und internationaler Ebene [6] wird die Verkehrskommission der SLgE die Richtlinien zu gegebener Zeit erneut überarbeiten.

\section{A. Allgemeine Richtlinien}

1. Bei einer aktiven Epilepsie ist die Fahrtauglichkeit in der Regel aufgehoben. Voraussetzungen für eine Erst- oder Wiederzulassung als Motorfahrzeuglenker sind eine dem Einzelfall angepasste periodische fachneurologi- sche Beurteilung sowie Überprüfung der Fahrtauglichkeit.

2. Eine Erst- oder Wiederzulassung als Motorfahrzeuglenker kann in der Regel erfolgen, wenn eine Anfallsfreiheit (mit oder ohne Antiepileptika) von einem Jahr besteht (Besonderheiten der verschiedenen Führerausweiskategorien siehe Abschnitt B).

Eine Verkürzung dieser Frist ist u.a. in folgenden Fällen möglich:

- einfache fokale Anfälle (ohne Bewusstseinsstörung) und ohne motorische, sensorische oder kognitive Beeinträchtigung beim Lenken;

- über mindestens 3 Jahre persistierende, ausschliesslich schlafgebundene Anfälle;

- Reflexepilepsien mit vermeidbarem auslösendem Stimulus.

Eine Verlängerung dieser Frist ist u.a. notwendig bei:

- Alkohol-, Medikamenten- oder Drogenabusus;

- fehlender Compliance bzw. Glaubwürdigkeit;

- Anfällen bei einer progressiven ZNS-Läsion;

- einer metabolischen Störung, die nicht ausreichend kontrollierbar ist;

- einer exzessiven Tagesschläfrigkeit (anfallsoder medikamentenbedingt).

3. Nach einem erstmaligen unprovozierten Anfall ist bei unauffälligen Befunden der erforderlichen neurologischen Diagnostik eine Fahrkarenz von 6 Monaten erforderlich.

Nach einem erstmaligen provozierten Anfall, einem posttraumatischen oder postoperativen Frühanfall (innerhalb einer Woche) sowie einem anderen Gelegenheitsanfall ist eine Fahrkarenz von 2 Monaten erforderlich.

Bei Patienten mit langjährigem bekanntem Krankheitsverlauf und mindestens 2jähriger Anfallsfreiheit kann bei einem isolierten Anfallsrezidiv eine 3monatige Fahrpause ausreichend sein.

4. Die EEG-Befunde müssen mit der Fahrtauglichkeit kompatibel sein.

5. Beim völligen Absetzen der Antiepileptika besteht für die Dauer des Absetzens des letz- 
ten Medikamentes und die ersten 3 Monate danach Fahruntauglichkeit. Ausnahmen sind in gutbegründeten Fällen möglich (insgesamt wenige Anfälle, Epilepsiesyndrome mit niedrigem Rezidivrisiko, erfolgreiche epilepsiechirurgische Behandlung, langsames Ausschleichen der Medikamente nach mindestens 3jähriger Anfallsfreiheit). Ist schon nach einem erstmaligen Anfall eine Behandlung erfolgt, kann beim langsamen Ausschleichen der Medikamente auf eine Karenz verzichtet werden. Bei den Kategorien C, C1, Taxi und D1 muss in solchen Fällen die Beurteilung der Karenzfrist durch den Fachneurologen erfolgen.

6. Ärztliche Aufklärungspflicht: Der behandelnde Arzt hat die betroffenen Patienten über diese Richtlinien $\mathrm{zu}$ informieren und seine auf den konkreten Einzelfall zutreffende Einschätzung der Fahrtauglichkeit zu erläutern. Diese Aufklärung sollte in den Patientenunterlagen dokumentiert sein. Eine gene-

\begin{tabular}{|l|l|}
\hline $\begin{array}{l}\text { Fachärztlich-Neurologisches Zeugnis zu Händen des zuständigen } \\
\text { Strassenverkehrsamtes/Vertrauensarztes }\end{array}$ \\
\hline Kraftfahreignung & $\begin{array}{l}\text { Name: } \\
\text { Geburtsdatum: } \\
\square \text { Erstzeugnis }\end{array}$ \\
und Epestätigungszeugnis
\end{tabular}

1. Diagnose?

2. Aktuelle Antiepileptika-Therapie?

3. Verlauf in den letzten 2 Jahren oder seit der letzten Berichterstattung vom?

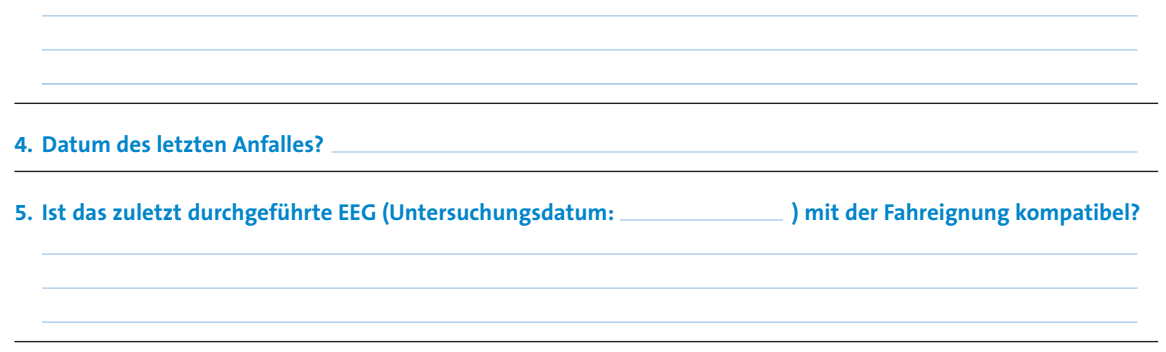

6. Besonderheiten bezüglich Compliance, Begleiterkrankungen, Suchtleiden?

7. Ist die Fahreignung aus neurologischer Sicht gegeben?

\begin{tabular}{l}
\hline \\
\hline 8. Die nächste Kontrolle mit Zeugniserstattung ist vorgesehen in \\
$\square 1$ Jahr \\
$\square 2$ Jahren \\
$\square$ anderer Zeitpunkt, nämlich \\
Begründung: \\
\hline \\
\hline
\end{tabular}


relle ärztliche Meldepflicht besteht nicht, hingegen ein Melderecht bei uneinsichtigen Patienten (Artikel 14, Absatz 4 SVG).

7. Meldepflicht des Patienten: Bei Auftreten eines Anfalles sofortiges Einstellen des Fahrens und Meldung an den behandelnden Arzt.

8. Die Ausstellung der Erstzeugnisse und der Bestätigungszeugnisse (Vorlage in der Anlage) betreffend Fahrtauglichkeit erfolgt gemäss den Weisungen der kantonalen Strassenverkehrsämter. Die Beurteilung der Kontrollfristen erfolgt durch den Neurologen.

\section{B. Besondere Bestimmungen bezüglich der einzelnen Führerausweiskategorien}

\section{Personenwagen (Kat. B und B1)} und Motorräder (Kat. A und A1)

$\mathrm{Zu}$ - und Weiterbelassung gemäss den allgemeinen Richtlinien.

\section{Lastwagen (Kat. C und C1) und Taxi} (berufsmässiger Personentransport) und Kleinbusse (Kat. D1)

Die Erst- oder Wiederzulassung zur Führerausweiskategorie C oder D1 ist bei einer einmal manifest gewesenen Epilepsie nur möglich, wenn eine 5jährige Anfallsfreiheit ohne Medikation besteht.

Bei einem erstmaligen, unprovozierten oder provozierten Anfall ist eine Karenzfrist von 2 Jahren einzuhalten. Wurden nach einem erstmaligen Anfall Antiepileptika gegeben, obliegt die Beurteilung der Karenzfrist dem Fachneurologen.

Ausnahme: wird bei C1 das Fahrzeug wie ein Privatfahrzeug benutzt (analog Kat. B), gelten die Bestimmungen von Kat. B.

\section{Car/Bus (Kat. D)}

Die Erst- oder Wiederzulassung zur Kat. D ist bei einer einmal manifest gewesenen Epilepsie nicht möglich. Nach einem erstmaligen unprovozierten oder provozierten Anfall ist eine Zulassung nur möglich, wenn eine 5 jährige Anfallsfreiheit ohne Medikation besteht.

4. Motorfahrzeuge mit Höchstgeschwindigkeit bis 45 km/h (Kat. F), Landwirtschaftliche Motorfahrzeuge (Kat. G), Motorfahrräder (Mofa) und Pistenfahrzeuge

Die Erst- und Wiederzulassung erfolgt gemäss den allgemeinen Richtlinien. Ausnahmen (insbesondere Verkürzung der Karenzfrist) sind in begründeten Einzelfällen möglich.

\section{Fahrlehrer und Experten}

Es gelten die Richtlinien der massgeblichen Führerausweiskategorien.

\section{Sonderfälle}

Tramwagenführer, Lokomotivführer, Piloten: Bei einer einmal manifest gewesenen Epilepsie oder auch nach einem erstmaligen provozierten oder unprovozierten Anfall besteht grundsätzlich Fahr- und Fluguntauglichkeit.

Bei Hubstaplerfahrern, Ballonführern, Baggerund Kranführern, Motorbootfahrern, Luftseilbahn- und Bergbahnführern erfolgt die Beurteilung der Fahrtauglichkeit gemäss den allgemeinen Richtlinien.

\section{Literatur}

1 Medici V, Bonetti C, Despland PA, Krämer G, Maag F, Markhoff R, et al. Epilepsie und Fahrtauglichkeit. Neue Richtlinien, erarbeitet von der Verkehrskommission der Schweizerischen Liga gegen Epilepsie (SLgE). Schweiz Ärztezeitung 1995; 76:1882-4 (versione italiana 1887-9).

2 Lewrenz H (Bearbeiter). Begutachtungs-Leitlinien zur Kraftfahrereignung des Gemeinsamen Beirats für Verkehrsmedizin beim Bundesminister für Verkehr, Bau- und Wohnungswesen und beim Bundesministerium für Gesundheit. 6. Auflage. Berichte der Bundesanstalt für Strassenwesen (Reihe «Mensch und Sicherheit»). Bremerhaven: Wirtschaftsverlag NW, Verlag für neue Wissenschaft; 2000.

3 Krämer G. Epilepsie und Führerschein: Neue Begutachtungs-Leitlinien. Aktuelle Neurologie 2000;27:90-2.

4 Fisher RS, van Emde Boas W, Blume W, Elger C, Genton P, Lee P, Engel J, Jr. Epileptic seizures and epilepsy: definitions proposed by the International League Against Epilepsy (ILAE) and the International Bureau for Epilepsy (IBE). Epilepsia 2005;46:470-2 (deutsche Übersetzung: Epileptologie $2005 ; 22: 84-7)$.

5 Beghi E, Sander JW. Epilepsy and driving (editorial). BMJ 2005;331:60-1.

6 Ben-Menachem E. Toward a more pragmatic view of driving and epilepsy. Epilepsy Current 2004; 4:133-4. 